\title{
Research on Sentimental Analysis Techniques
}

\author{
V. Senthil Kumar, B.Vinoth Kumar
}

\begin{abstract}
The sentimental analysis may be a dominant role in opinion mining is in addition stated as sentiment analysis due to clear sort, review sites, blogs, and tweets square measure on the market in digital sort. Sentiment analysis is that the sphere of study that analyses consumer opinion, feedback, sentiment analysis, attitudes and feeling from communication. At intervals fraction second, we've got an inclination to classify the text in many manner in several seconds. It's one all told the active analysis areas in communication method. There are a unit vary of techniques we would like to classify the opinion reviews. the most problematic within the sentiment analysis is to grasp the usage of negation and also the taxonomy of positive and negative sentiments recorded by the users in the group. the most aim of this paper boon a survey relating to the presently accessible technique, application and drawback that seem within the field of opinion mining.
\end{abstract}

Index terms- Sentiment analysis, Navies Bayes,Support vector machine, Aspect extraction, Positive and negative.

\section{INTRODUCTION}

The sentiment is an feeling or feeling in the patron's comments to be analysis in statistics assortment approach and additionally the approach to extract and capture data for analysis in language approach and linguistic rely evaluation its accustomed extract and analyze information from Net order to renowned the subjective opinion of a file or the assortment of files, like weblog posts, reviews, information articles and social media feeds like tweets and standing updates Sentiment Classification goals at mining the planet Wide net textual content of product critiques with the aid of clients to classify the evaluations into fantastic or bad critiques. Device-managed opinion mining from the opinions is beneficial to each clients and sellers. Classification may well be a project that want to be addressed on the facet of the sentiment evaluation disadvantage. The text matters might probably or may not contain useful reviews or comments. The subjective sentences unit of dimension the applicable textual content, and in addition the goal sentences unit the orthogonal text. We have a propensity to tend to generally tend to need to arrange the sentences that unit helpful or now not. The subjective sentences unit these sentences having beneficial records for the sentiment analysis. Such class is termed as subjective category. Some works are in dire straits that focus on this specific disadvantage. A number of the authors gift a way of subjective identity for sentiment evaluation. Typically this could be generally vital as a outcomes of the orthogonal records from the reviews will be eliminated. This excludes the strategy overheads of an large amount of matter statistics. The strategy they propose to apply a minimum

Revised Manuscript Received on 14 August, 2019.

V.Senthil Kumar, Assistant Professor/CSE, Kumaraguru College of Technology, Coimbatore, Tamilnadu, India. (Email: senthilkumar.v.cse@kct.ac.in)

B.Vinoth Kumar, Associate Professor/IT, PSG College of Technology, Coimbatore, Tamilnadu, India. (Email: bvk@cse.psgtech.ac.in) pass supply subjective extracts from the textual content. The paintings is focused some of the sentence degree subjective extraction.

A class method victimization Naive theorem classifier is employed in They gift the consequences of growing subjective classifiers victimization un-annotated texts for paintings. All through this work for learning Subjective and Objective sentences, the method mechanically generates a training statistics. Typically, typically executed by a Ruleprimarily based technique. The rule-based subjective classifier classifies a sentence subjective if it includes 2 or further sturdy subjective clues. In distinction, the guidelinebased totally goal classifier search for the absence of clues it classifies the sentence is objective if there do no longer appear to be any strong subjective clues among this sentence, there's at the foremost one robust subjective trace the preceding and subsequent sentence blended, and on the foremost 2 weak subjective clues a few of the modern, previous, and next sentence combined classifiers. They use Subjective reality, Subjective Recall, Subjective F live, Objective accuracy, Objective Recall and Objective F stay for the evaluation. They alongside put in force a selfeducation procedure for the system. The device learning, function opportunity is that the employment of unique variables or facts factors to maximise efficiency all through this kind of superior technological know-how. Feature alternative is assumed as variable opportunity, attribute alternative or set opportunity. Feature preference is Absolutely completely specific from belongings discount. Every strategy is want to trim the quantity of attributes at durations the dataset, however a assets reduction technique will so by means of growing new combos of attributes, while the characteristic alternative approach embodies and exclude attributes gift at periods the information while now not dynamic them. Feature alternative method on your assignment to create an accurate prognostic model. By choosing selections in order to provide you with with nearly almost as exact or higher accuracy whereas requiring much less data. The feature opportunity methods are accustomed establish and subtract inessential, immaterial and redundant attributes from data that don't make a contribution to the accuracy of a prognostic model or may basically lower the accuracy of the model.

\subsection{Filter methodology}

Filter ways that unit of dimension now and again used as a pre-processing step. The selection of choices is freelance of any gadget studying formula. Instead, decisions unit of size elite on the idea of their rankings in numerous arithmetic exams for his or her correlation with the top end 
result variable. The correlation can be a subjective term here. For fundamental guidance, you will be ready to see the subsequent table for outlining the correlation.

\subsection{Wrapper technique}

In wrapper strategies, we have got AN inclination to adopt and use a bunch of choices and teach a model pattern them. Supported the inferences that we've got AN inclination to draw from the previous model, we've were given AN inclination to arrange to feature or exclude decisions out of your set. Is basically reduced to a pursuit drawback. These strategies region unit usually computationally terribly treasured. Some commonplace samples of wrapper strategies region unit forward function one-of-a-kind, backward characteristic removal, algorithmic function removal, and so on.

\section{-Forward Selection:}

Forward desire is AN unvarying approach amongst that we've got were given AN inclination to start off with having no feature some of the model. In each generation, we've got were given $\mathrm{AN}$ inclination to remain including the function that exceptional improves our model until AN addition of a completely unique variable will not improve the overall performance of the version.

\section{- Backward Elimination:}

In backward elimination, we've were given a tendency to start off with all the alternatives and eliminates the tiniest quantity crucial function at each new release that improves the overall performance of the version. We've got a tendency to copy this until no improvement is set on elimination of selections.

\section{- Algorithmic Feature elimination:}

it is a grasping development rule that objectives to hunt out the handiest participating in feature set. It again and again creates fashions and keeps apart the sole or the worst taking part in function at each iteration. It constructs succeeding model with the left selections till all of the options ar exhausted. It then ranks the options supported the order in their removal.

\subsection{Embedded technique}

Embedded methodology combine the features' of filter and wrapper method. It's applied with the aid of algorithms that have their personal intrinsical feature choice technique.
Some of the foremost commonplace samples of those techniques are LASSO and RIDGE regression which have built-in penalty carry out to reduce over becoming. Lasso regression perform L1 regularization that provides penalty adore quantity of the magnitude of constant. Ridge regression plays L2 regularization that provides penalty much like sq. Of the value of constant.

\section{LITERATURE REVIEW}

A deep neural community version combining convolutional neural network and nearby lengthy basic cognitive method (CNN-RLSTM) for the assignment of goal-primarily based sentiment evaluation [1]. The experimental consequences show that our CNN-RLSTM model performs beyond present techniques like SVM, attention primarily based definitely LSTM and multiattention based totally $\mathrm{CNN}$, that corroborate the effectiveness of this version. What's greater, the CNNRLSTM version fees alone [*fr 1$]$ time compares to the initial attention-based totally LSTM community model. The geo-sentiment evaluation contains 3 demanding situations discrepancy among a couple of sentiment classifiers, discrepancy most of the feelings of geo-neighbours, and dataset heterogeneity[2]. We will be predisposed to tend to projected a unique framework composed of three levels: sentiment evaluation, spatial-temporal partitioning, and geo sentiment modeling. The framework to investigate the failures of cyclone Sandy and cruciferous plant Earthquake supported datasets amassed from Twitter and Flickr. Random Forest classifier turned into accustomed establish positive, poor or impartial sentiment of particular file [8].The Random Forest components is closed scikit-learn (Random Forest makes use of numerous tree-based classifiers to form predictions, so the "woodland"). Below, we've a dishonest to line the variety of trees to a hundred as an low priced default price. Many bushes might (or won't) perform better, but will surely take longer to run. Likewise, the ton of choices you embody for each evaluate, the longer this will take. The mixture method of POS tagging, FCAprimarily based domain philosophy and SVM classifier shall beautify the sentiment class [10]. By exploitation this approach we will be predisposed to are equipped to browse the energy or we have a tendency tokens of the merchandise or objects thousands of detail which we are hoping ar useful for added 
International Journal of Engineering and Advanced Technology (IJEAT)

ISSN: 2249 - 8958, Volume-8, Issue-6S3, September 2019

\begin{tabular}{|c|c|c|c|c|c|}
\hline I & AUTHOR & TITLE & METHOD & ADVANTAGES & DIFADVAKTAGES \\
\hline 1 & $\begin{array}{l}\text { Pyan } \\
\text { Gen oho } \\
\text { Peng } \\
\text { Lanedou }\end{array}$ & $\begin{array}{l}\text { A Desp Neral Neteok } \\
\text { Model for Targetbased } \\
\text { Sertinent Analyst }\end{array}$ & 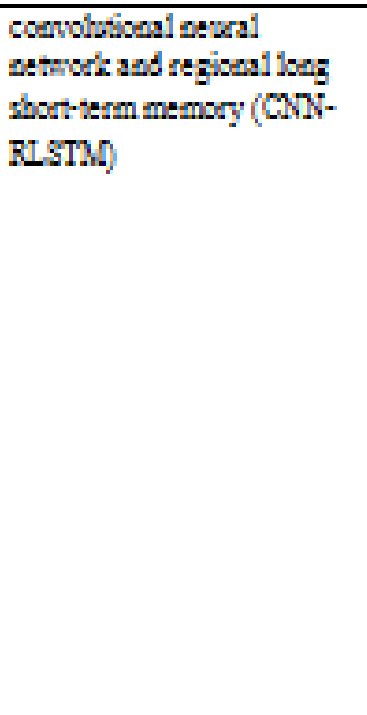 & 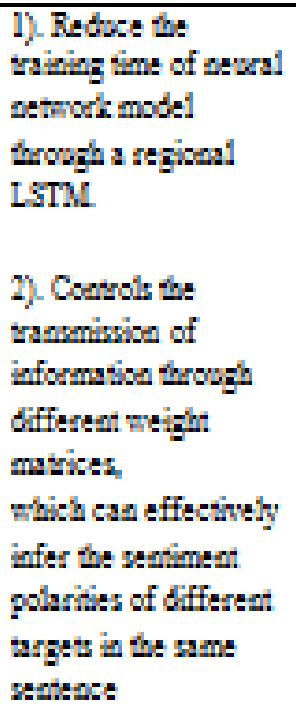 & 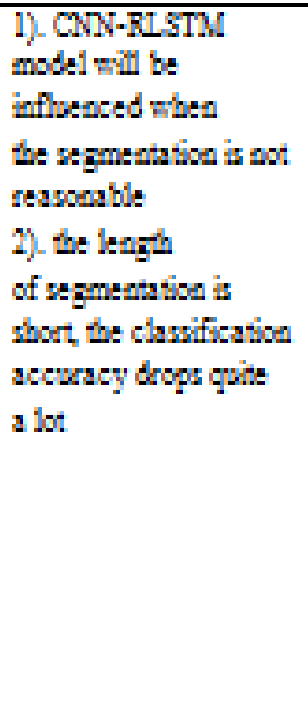 \\
\hline 2 & 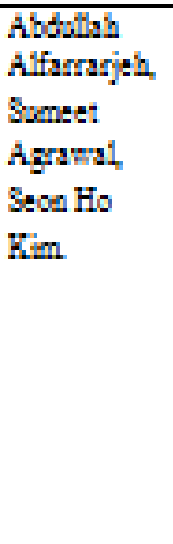 & 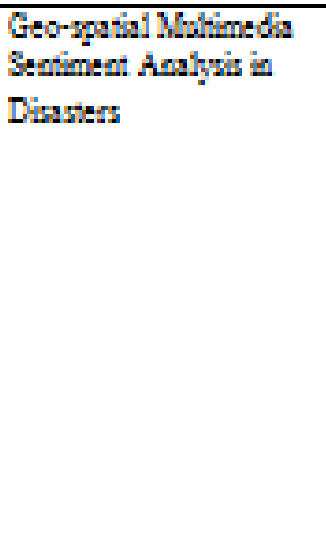 & 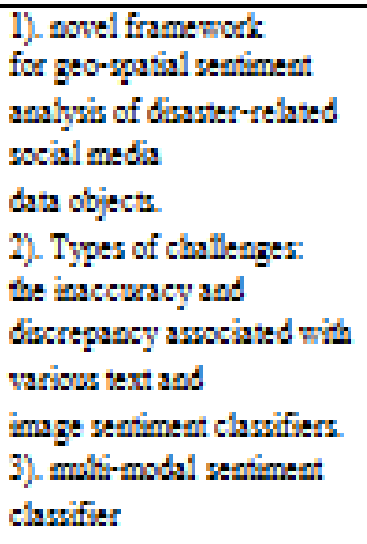 & 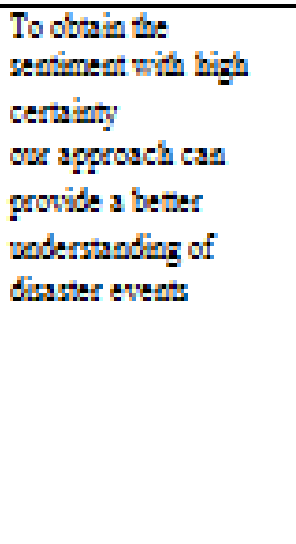 & $\begin{array}{l}\text { mitr-modal sentinent } \\
\text { cherifur } \\
\text { antors ou other } \\
\text { hagses }\end{array}$ \\
\hline 3 & 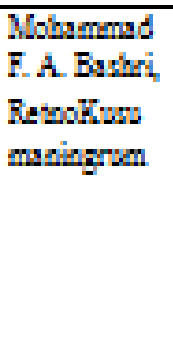 & 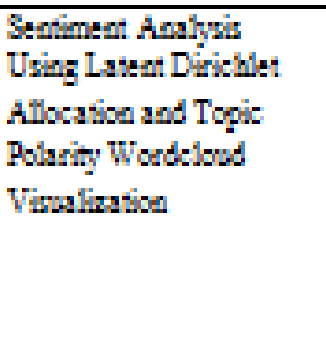 & 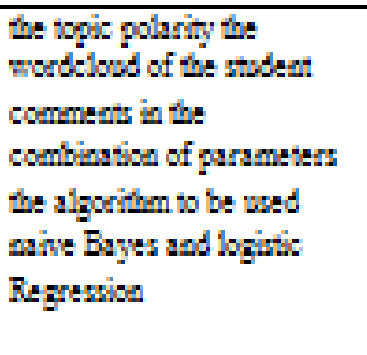 & 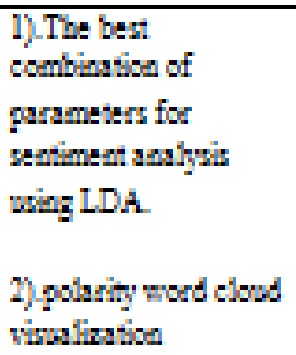 & 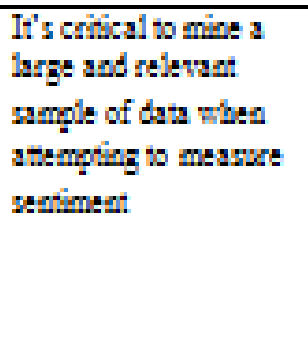 \\
\hline 4 & $\begin{array}{l}\text { Ravy lose } \\
\text { Warghese } 9 \\
\text { Chowall }\end{array}$ & 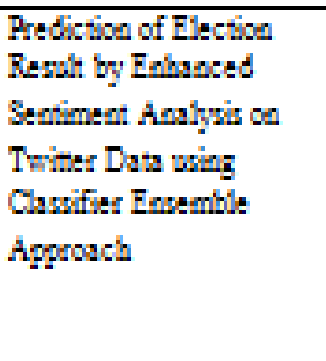 & 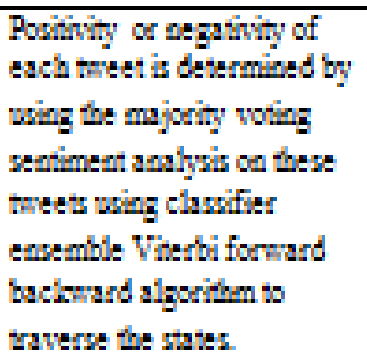 & 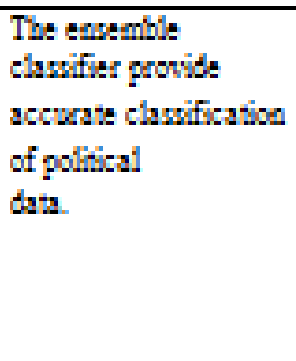 & 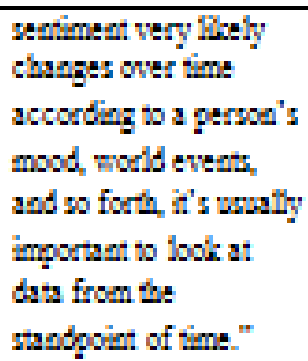 \\
\hline 3 & $\begin{array}{l}\text { Morihatian } \\
\text { abraj Bam } \\
\text { Mona } \\
\text { Reddy }\end{array}$ & 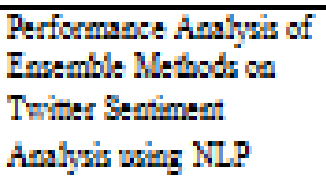 & 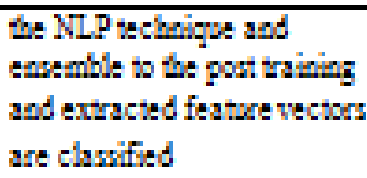 & 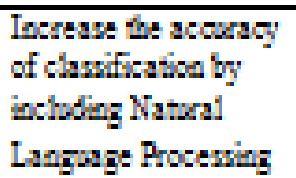 & 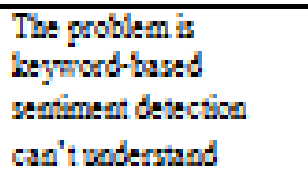 \\
\hline
\end{tabular}

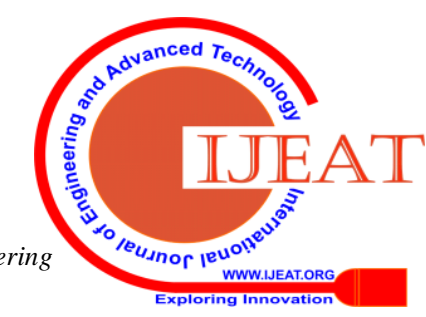




\begin{tabular}{|c|c|c|c|c|c|}
\hline & Goddet & Techanges & & Techoiges (NL) & 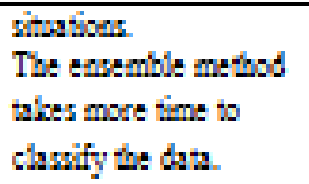 \\
\hline 6 & $\begin{array}{l}\text { Che Wou } \\
\text { Pak } \\
\text { Derkyoug } \\
\text { to }\end{array}$ & 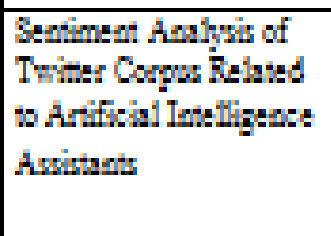 & 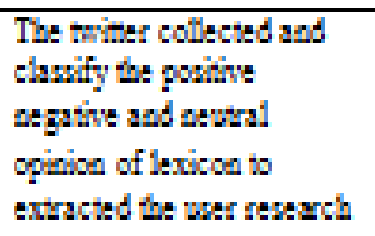 & $\begin{array}{l}\text { Arfinal instigeos } \\
\text { Awistant is } \\
\text { satutically beter. }\end{array}$ & $\begin{array}{l}\text { aatzal langage } \\
\text { protes as ow } \\
\text { optinized complesty }\end{array}$ \\
\hline 7 & $\begin{array}{l}\text { Shail And } \\
\text { Phandeow } \\
\text { a And } \\
\text { Phand }\end{array}$ & $\begin{array}{l}\text { Triwer Sectimeat } \\
\text { Clawifoation wing } \\
\text { Stanford NLP }\end{array}$ & 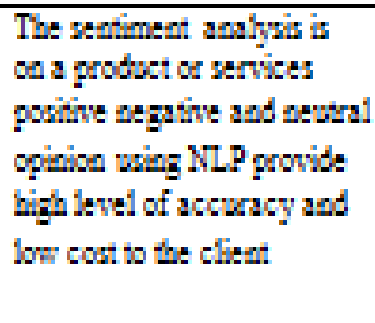 & 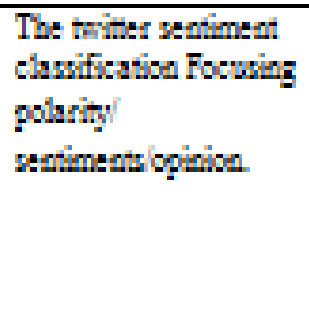 & 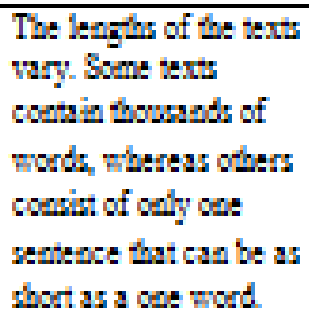 \\
\hline 5 & $\begin{array}{l}\text { S } \\
\text { Zharmagan } \\
\text { bew } \\
\text { Alexand }\end{array}$ & 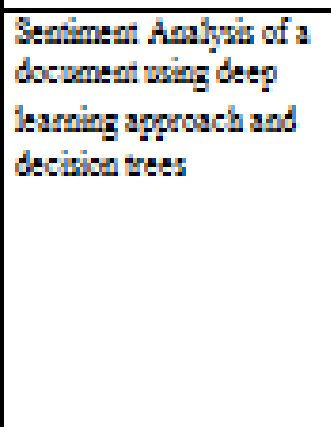 & 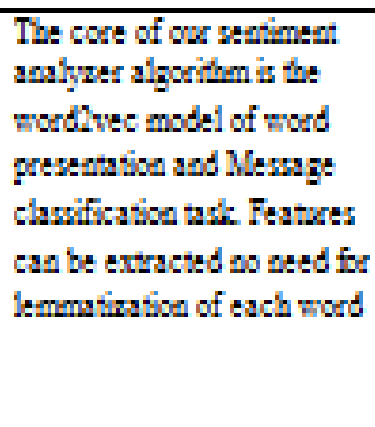 & 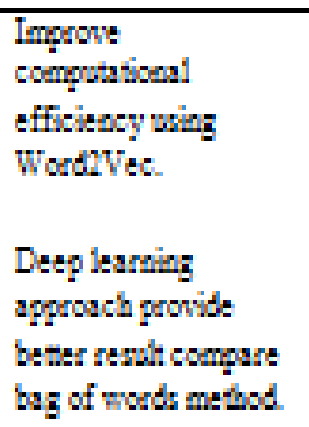 & 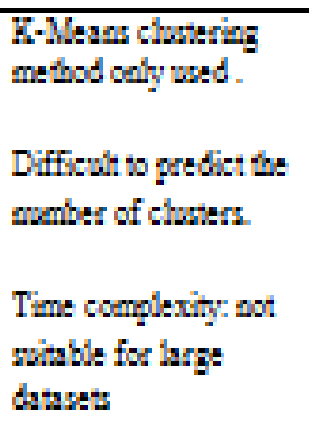 \\
\hline 9 & 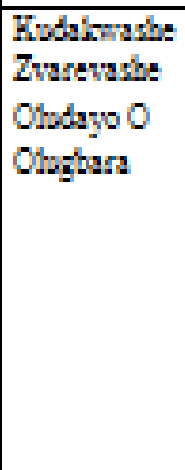 & 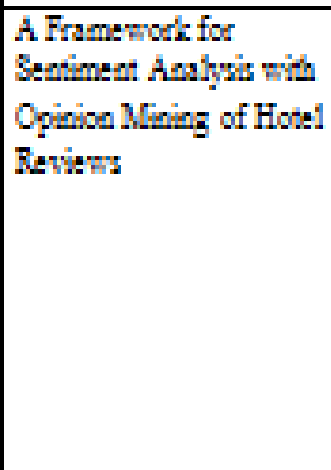 & 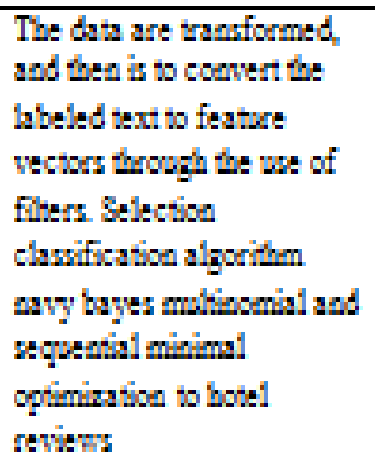 & 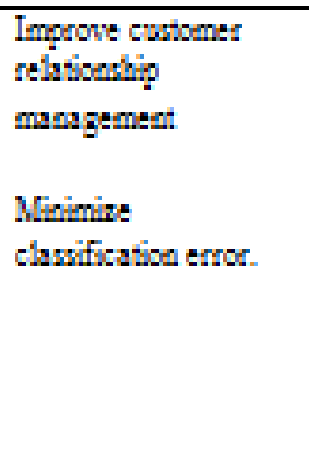 & $\begin{array}{l}\text { If's Only Fousing } \\
\text { botel resures }\end{array}$ \\
\hline 10 & $\begin{array}{l}\text { ThinPhy } \\
\text { Physesea }\end{array}$ & 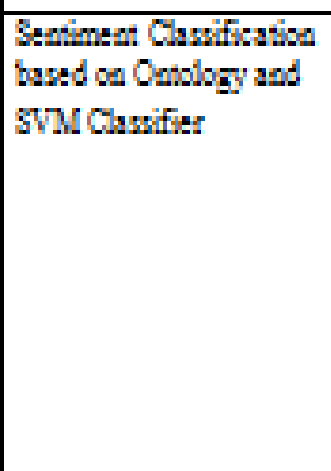 & 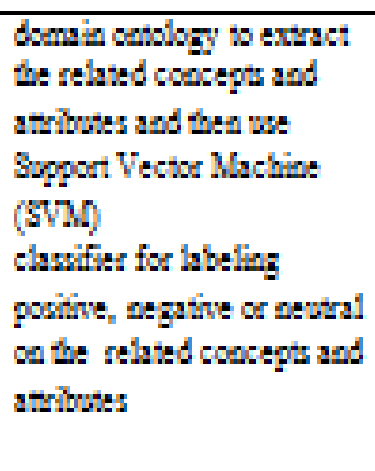 & 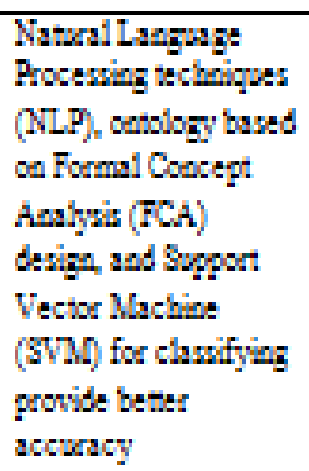 & 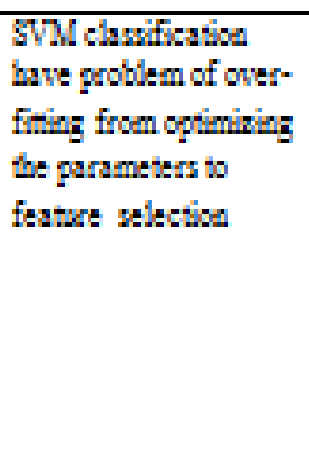 \\
\hline
\end{tabular}




\section{CHALLENGES FOR SENTIMENT ANALYSIS}

Sentiment analysis categorizes text as nice, poor or impartial, so it'll be called as text type undertaking like traditional textual content class and opinion classification. In textual content class it is many classes and lots of subjects but in sentiment analysis it's most effective three classes. There ar many issues that construct sentiment evaluation robust as compared to text type. The foremost challenges that ar confronted by using sentiment evaluation ar the following:

\subsection{Domain dependency}

The essential venture confronted by way of sentiment evaluation is that the area structured nature of sentiment phrases like, One choices set may also offer glorious overall performance in one domain, at a similar time it carry out virtually terrible in any other domain.

\subsection{Detection of spam and faux critiques}

The internet includes each real and spam contents. For powerful Sentiment category, this spam content material have to be compelled to be eliminated earlier than method. This might be achieved through extraordinary duplicates, by way of sleuthing outliers and by way of considering name of evaluation.

\subsection{Classification Filtering}

For better sentiment category end result this category must be forced to be reduced. The prospect of filter bubble offers inappropriate opinion sets and it results fake document of sentiment.

\subsection{Imbalance In Convenience Of Opinion Mining Software}

The opinion mining laptop code is pretty treasured and presently cheap only to huge companies and government. It is at the way aspect the common citizen's expectation. This is probably procurable to any or all or any little and medium size agencies at reasonably-priced worth, therefore each person receives have the good thing about it.

\subsection{Incorporation of Opinion With Implicit And Behaviour Records}

For booming sentiment evaluation, the opinion words must be forced to concatenate with implicit records. The implicit data seem to be the real behaviour of sentiment words.

\subsection{Tongue System Language}

The language overhead like ambiguity, co-reference, unclearness, cerebration and so on. Created quandary in sentiment evaluation too.

\section{SUBJECTIVITY/OBJECTIVITY}

\subsection{Classification}

Classification is probably a undertaking that have to be compelled to be addressed beside sentiment analysis downside. The text things may additionally or might not contain useful critiques or feedback. The subjective sentences ar the relevant texts, and additionally the target sentences are the digressive texts. So we will be inclined to have a tendency to should depicted the sentences that are helpful for U.S. And folks that are not. The subjective sentences ar the ones sentences having helpful records for the sentiment analysis. Such type is named as sound judgment classification. Some works are carried out that specialise in this unique downside. Within the authors present a way of sound judgment identity for sentiment evaluation [1]. Generally this will be often important as a consequences of the beside the point statistics from the critiques is likewise removed. This excludes the method overheads of companion diploma outsized quantity of depend information. The strategy they suggest is exploitation minimum cuts to deliver subjective extracts from the textual content. The paintings has been focused at periods the sentence stage sound judgment extraction.

A classification technique exploitation Naive theorem classifier is used in [2]. They present the effects of growing subjectivity classifiers exploitation un-annotated texts for employment. Throughout this paintings of gaining knowledge of Subjective and Objective sentences, the course of action routinely generates employment records. This may be carried out by means ofa Rule-based method. The rule-primarily based subjective classifier classifies a sentence as subjective if it contains or in addition sturdy subjective clues. In distinction, the rule-based totally goal classifier look for the absence of clues: it classifies a sentence as objective if there aren't any robust subjective clues interior this sentence, there is at the most one sturdy subjective trace the previous and next sentence combined, and at the most two weak subjective clues interior this, preceding, and subsequent sentence combined classifiers. They use Subjective accuracy, Subjective Recall, Subjective F live, Objective accuracy, Objective Recall and Objective F stay for the analysis. They similarly put into effect a self employment method for the device.

\section{V.APPROACHES\& RESULTS}

\subsection{MACHINE LEARNING APPROACHES}

Machine gaining knowledge of may be a subfield of computing that aims at making the laptop capable of work whereas not being programmed expressly. Machine learning peruse records to journey looking for styles and makes use of them to reinforce the packages knowledge consequently the program can develop and modify in step with the information it is uncovered to numerous device gaining knowledge of strategies tried to classify opinions:

$\square$ Naïve Thomas Bayes classifier

$\square$ maximum entropy classifier

$\square$ Support vector machine

\subsection{LEXICON BASED APPROACHES}

Lexicon based technique assumes the linguistics orientation of the entire textual content is able to the add of character linguistics orientation of words and terms. Lexicon technique makes use of a e-book of records of words beside their numerous linguistics orientation. The e-book of information are going to be created manually or 
mechanically exploitation seed phrases. Commonly the adjective words ar accustomed establish he linguistics orientation of the textual content. Initial all of the adjective phrases ar extracted from the text and their numerous linguistics orientation values are extracted. These values are then accustomed calculate the very last polarity of the textual content.

\subsection{APPLIED MATHEMATICS APPROACHES}

Statistical analysis typically includes aggregation and analyzing data samples to seem at tendencies or styles. Carried out arithmetic version considers the understanding to be a combination of elements and rankings and therefore the motive is to extract the components from the information and to pick out and classify the feelings into scores. A word taking place loads of commonly in high-quality texts is taken beneath consideration to own a superb polarity while a word whose prevalence is similarly frequent in poor texts is taken under attention terrible. A phrase with comparable frequency of occurrence is taken underneath attention impartial.

\subsection{SUPERVISED AND UNSUPERVISED LEARNING}

\subsubsection{Supervised Learning}

In supervised gaining knowledge of the gadget is educated exploitation output datasets so on get hold of the desired end result. In supervised gaining knowledge of the kinds the knowledge is assigned to is believed earlier than computation. The version commonly defines the impact one set of observations put on any other set of observations.

\subsubsection{Unsupervised Learning}

In unsupervised studying the knowledge is clustered in lessons and rule is fashioned to differentiate. The version isn't always given the perfect consequences throughout the employment. Latent variables ar the explanation for all observations. Since supervised studying attempts to hunt out a correlation [20] between a pair of units of statistics it's further handy to use unattended gaining knowledge of for fashions with deep hierarchies. With unattended studying time can boom linearly in type of tiers of version hierarchy considering that learning can continue from one level to a wonderful and most effective one step is needed at a hierarchy stage.

Marketing are expecting Zhang et al. [7] projected weak point realizeer system that may facilitate producers word their product weak spot from Chinese evaluations by means of exploitation factors based sentiment analysis. There place unit a few enterprise and loose sentiment evaluation offerings location unit to be had, Radiant6,Sysomos, Viral heat, Lexalytics, and so forth. Arebusiness.

\section{APPLICATIONS OF SENTIMENT ANALYSIS}

Sentiment evaluation could also be utilised in varied fields for numerous capabilities. This section discusses style of the common ones. The examples conferred at some point of this section do not seem like entire but just a snap shot of the opportunities.

\subsection{ON-LINE COMMERCE}

The most preferred use of sentiment evaluation is in etrade sports. Websites lets in their customers to put up their enjoy regarding searching out and merchandise characteristics. They offer outline for the merchandise and completely exclusive selections of the merchandise via distribution rankings or scores. Customers can simply experiment opinions and recommendation records on entire product moreover as unique product selections. Graphical outline of the general product and its choices is given to consumer. Common capitalist web sites like amazon.Com affords overview from editors and besides from clients with score statistics. Http://tripadvisor.In could also be a standard computer that gives evaluations on inns, journey locations. They incorporate seventy five millions reviews and evaluations international. Sentiment analysis enables such websites by means of dynamical discontent customers into promoters by way of analyzing this massive extent of evaluations.

\subsection{VOICE OF THE MARKET (VOM)}

Voice of the Market is concerning decisive what clients unit of measurement feeling concerning products or services of competitors. Accurate and well timed information from the aggressive advantage and goal time. This

\subsection{VOICE OF THE CUSTOMER (VOC)}

Voice of the purchaser is concern concerning what or offerings patron enjoy new opportunities for product inventions. Extracting consumer evaluations to boot enables verify practical desires of the products and a number of nonpurposeful desires like overall performance and worth.

\subsection{WHOLE NAME MANAGEMENT}

Brand name Management is problem regarding managing your call in market. Opinions from clients or the opposite parties can harm or decorate your call. Whole call Management (BRM)consumer. Currently online at a high charge. That produces opportunities for companies to manage and give a boost to complete name. Presently entire belief is determined not completely by way of marketing, promotion and organization electronic communique. Brands vicinity unit currently a add of the conversations concerning them. Sentiment analysis enables in decisive however organisation's complete, products or services is being perceived by using community on line.

\subsection{GOVERNMENT}

Sentiment evaluation enables government in assessing their strength and weaknesses by analysing reviews from public. As an example, "If this can be the kingdom, but do you anticipate fact to return back out? The MP WHO is investigation $2 \mathrm{~g}$ rip-off himself is deeply corrupt." [15].This case in reality suggests poor sentiment concerning authorities. Whether or not or now not it's following citizens' reviews on a new108 device, feature strengths accomplice degreed weaknesses all through an accomplishment campaign in authorities job, assessing the fulfilment of the digital submission of tax returns, or many 
one-of-a-kind regions, we're going to see the potential for sentiment evaluation.

\section{INFERENCE}

A deep neural community version combining convolutional neural community and regional lengthy reminiscence (CNN-RLSTM) for the assignment of targetprimarily based sentiment evaluation. To induce the sentiment with excessive actuality, the model measures the war of words among connected sentiment labels either via entropy or variance metric.

The deep getting to know approach tough in place of clean strategies as "bag-of-words", it shows simplest slightly higher results. It's attending to be due to mistakes degree in words' clump and noises that regarded in preprocessing. POS tagging, FCA-based area philosophy and SVM classifier can decorate the sentiment category.

\section{CONCLUSION}

This paper makes a trial to provide a survey present techniques for opinion mining in rule-based totally approach and lexicon-primarily based processes with some analysis metrics. The overall performance of system mastering ways, like SVM and naive mathematician have the upper accuracy and may be concept of the baseline mastering approaches that, while lexicon-primarily based approaches that location unit very effective in a few cases, that need few efforts at some stage in a human-labelled document. The rule based technique is extraordinarily passionate to the guideline method for performance, therefore mainly this technique underperforms in difference with machine getting to know and lexicon method. The take a look at else shows that a tremendous deal of the cleaner info, a great deal of accurate results vicinity unit generally received. It location unit typically administrated for higher analysis methods that at some point of this space, however due to the linguistics by way of considering better rule definition to strengthen rulebased technique. Many of the planet of internet, most people of people depend upon social networking web sites to urge their valued info, studying the evaluations from these blogs can yield a far higher information and facilitate of their selection-making.

\section{REFERENCES}

1. B. Liang, Q. Liu, J. Xu, Q. Zhou, P. Zhang, AspectBased Sentiment Analysis based on Multi-Attention CNN," Journal of Computer, Research and Development. Chinese,vol.54, No. 8, pp. 1724-1735, 2017.

2. J. Yuan, S.Mcdonough, Q.You, and J. Luo. "Sentribute: image sentiment analysis from a mid-level perspective." In Proceedings of the Second International Workshop on Issues of Sentiment Discovery and Opinion Mining, p. 10. ACM, 2013.

3. P. Aliandu, "Sentiment Analysis on Indonesian Tweet", in The Proceedings of The 7th ICTS, Bali, 2013, pp. 203 $-208$.

4. Farhan Hassan Khan, Saba Bashir, Usman Qamar, TOM: Twitter opinion mining framework using hybrid classification scheme Decision Support Systems 57 (2014) 245257, 2013 Elsevier B.V.

5. C. Hung and H.-K. Lin, "Using objective words in sentiwordnet to improve sentiment classification for word of mouth," IEEE Intelligen Systems, p. 1,2013.
6. A. Pak and P. Paroubek, "Twitter as a corpus for sentiment analysis and opinion mining," in LREc, vol. 10, May. 2010, pp. 1320-1326.

7. VarshaSahayak, VijayaShete, ApashabiPathan, "Sentiment Analysis on Twitter Data", International Journal of Innovative Research in Advanced Engineering(IJIRAE),Issue 1, Volume 2 (January 2015) Page -178

8. M.F. Caropreso, S. Matwin, F. Sebastiani, "A learnerindependent evaluation of the usefulness of statistical phrases for automated textcategorization," Text databases and document management: Theory and practice, 2001, pp. 78-102.

9. E. Cambria, B. Schuller, Y. Xia and C. Havasi, "New avenues in opinion mining and sentiment analysis". IEEE Intelligent Systems, vol. 28, no. 2, pp. 15-21, 2013.

10. R. Mukras., J. Carroll. A comparison of machine learning techniques applied to sentiment classification, 2004.

11. M. D. Devika, C. Sunitha, and A. Ganesh, "Sentiment Analysis: A Comparative Study on Different Approaches," in Procedia ComputerScience, 2016, vol. 87, pp. 44-49.

12. Z. Hu, J. Hu, W. Ding, and X. Zheng, "Review Sentiment Analysis Based on Deep Learning," in 2015 IEEE 12th International Conference on e-Business Engineering, 2015, pp. 87-94.

13. D. M. E.-D. M. Hussein, "A survey on sentiment analysis challenges,” J. King Saud Univ - Eng. Sci., vol. 34, no. 4, 2016.

14. S. K. Dwivedi and B. Rawat, "A review paper on data preprocessing: A critical phase in webusage mining process," in 2015 International Conference on Green Computing and Internet of Things (ICGCIoT), 2015, pp. 506-510.

15. C. Bhadane, H. Dalal, and H. Doshi, "Sentiment analysis: Measuring opinions," Procedia Comput. Sci., vol. 45, no. C, pp. 808-814, 2015.

16. Q. Rajput, S. Haider, and S. Ghani, "Lexicon-Based Sentiment Analysis of Teachers ' Evaluation," Hindawi Appl. Comput. Intell. Soft Comput., vol. 2016, no. 6, 2016.

17. R. Nithya and D. Maheswari, "Sentiment analysis on unstructured review," in IEEE Proceedings - 2014 International Conference onIntelligent Computing Applications, ICICA 2014, 2014, pp. 367-371.

18. K. Ahmed, N.El Tazi, and A. H. Hossny, "Sentiment Analysis over Social Networks: An Overview," in 2015 IEEE International

19. Conference on Systems, Man, and Cybernetics (SMC), 2015, no. October, pp. 2174-2179

20. V.Senthil Kumar,B.Vinoth Kumar and P.Saranya, "Normalized page count and text based metric for computing Semantic similarity between web documents," in Journal of Advanced Research in Dynamical and Control Systems- 2017 Vol. 9. Sp- 6 pp. 1865-1875. 